\title{
La economía de la salud en México
}

\author{
Leonardo Lomelí Vanegas
}

\section{Resumen}

La crisis sanitaria mundial provocada por la pandemia de la enfermedad por coronavirus (COVID-19) ha puesto de manifiesto la importante relación que existe entre la economía y la salud. Esta relación se da en los planos microeconómico, macroeconómico e institucional, ya que los mercados de la salud tienden a sufrir fallas de mercado; el gasto sanitario tiende a aumentar como porcentaje del producto interno bruto (PIB), y su financiamiento tiene consecuencias en las finanzas públicas a largo plazo. En el caso de México, la creación de un sistema de salud público segmentado, como consecuencia del sistema de protección social que se viene aplicando en el país desde 1943 y que ha sido objeto de diversas reformas en los últimos años, ha contribuido a convertir el acceso a la salud en un importante factor de desigualdad, que repercute en la pobreza multidimensional de un segmento significativo de la población.

\section{Palabras clave}

Salud, economía de la salud, salud pública, gastos públicos, servicios de salud, derecho a la salud, población, bienestar social, política sanitaria, México

\section{Clasificación JEL}

H41, H44, I10, I13, I14, I18, I38

\section{Autor}

Leonardo Lomelí Vanegas es Profesor Titular de la Facultad de Economía de la Universidad Nacional Autónoma de México (UNAM). Actualmente es Secretario General de la UNAM. Correo electrónico: leolomeli@gmail.com. 


\section{Introducción ${ }^{1}$}

La pandemia de la enfermedad por coronavirus (COVID-19), provocada por el virus SARS-CoV-2, ha puesto de manifiesto la importancia de las fortalezas y debilidades de los sistemas de salud de cada país, así como el carácter oligopólico o monopólico de los principales mercados relacionados con la salud, la insuficiente capacidad regulatoria de los Estados a nivel nacional y la ausencia de una gobernanza mundial capaz de articular una cooperación eficiente en situaciones como la que se enfrenta actualmente. Por todo lo anterior, no resulta exagerado afirmar que la economía de la salud se ha convertido en un área estratégica, no solo en lo que respecta al análisis económico, sino también al bienestar general y la seguridad mundial.

La economía de la salud nació como una rama de la economía del bienestar. Eso explica que buena parte de la bibliografía disponible aborde esta esfera de estudio desde una perspectiva microeconómica y, en consecuencia, se centre en analizar los mercados de prestación de servicios sanitarios, producción de insumos y tecnología para los tratamientos y, más recientemente, en proponer criterios para la asignación de recursos a los distintos procedimientos de la medicina (Martínez, 2014). Desde una perspectiva microeconómica, una preocupación fundamental de la economía de la salud radica en corregir las fallas del mercado a la hora de producir dichos bienes y servicios. A su vez, esto implica distintas formas de intervención del Estado en esos mercados; desde la provisión directa hasta la regulación, pasando por el subsidio directo o indirecto de la producción o el consumo.

No obstante, la economía de la salud también tiene una dimensión macroeconómica: el gasto en salud como porcentaje del producto interno bruto (PIB) o del producto nacional bruto (PNB) (según el país que se analice) tiende a incrementarse con el paso del tiempo, y el financiamiento de los servicios sanitarios constituye una de las principales preocupaciones de los gobiernos en la actualidad. Además, la salud es una variable que influye en el crecimiento económico a través del capital humano. Al mismo tiempo, el acceso a los servicios de salud y su calidad se convierten en variables fundamentales del bienestar de la población, e inciden tanto en la distribución de los ingresos como en los niveles de pobreza multidimensional. Por lo tanto, la relación entre la salud, el desempeño económico y el bienestar social es estratégica y constituye una de las áreas de análisis más prometedoras para las teorías modernas de desarrollo. La experiencia de las últimas décadas avala la tesis de Angus Deaton de que el acceso a la salud, además de ser una variable muy importante para explicar el desarrollo, es un factor clave para reducir o ampliar la desigualdad (Deaton, 2015).

\section{La macroeconomía de la salud}

La economía de la salud tiene una dimensión macroeconómica cada vez más importante. En todos los países, el gasto en salud tiende a incrementarse a largo plazo, como consecuencia de dos transiciones que guardan una estrecha relación entre sí y con los respectivos niveles de desarrollo, a saber: la transición demográfica y la transición epidemiológica. El resultado combinado de ambas es que la población vive más años y una mayor proporción de ella padece enfermedades crónico-degenerativas, situaciones que traen consigo un alza sostenida en los costos de la atención sanitaria, lo que a su vez provoca un aumento del gasto en salud a largo plazo.

La transición demográfica se caracteriza por el envejecimiento paulatino de la población a raíz del incremento de la esperanza de vida y los cambios en los patrones reproductivos, que conducen a su vez a modificaciones en la estructura de edades, como se observa en la pirámide demográfica. El

1 Se agradecen la ayuda y los valiosos comentarios de Ismael Valverde Ambriz. 
aumento de la esperanza de vida se debe a la difusión de medidas de higiene que reducen la propagación de ciertas enfermedades y a la universalización de los tratamientos que las curan. Los cambios en los patrones reproductivos obedecen a la creciente incorporación de la mujer al mercado laboral y a la planificación familiar; en la actualidad, las familias optan por tener menos hijos, tenerlos a edades más tardías o no tener descendencia. El aumento de las tasas de escolarización ha reforzado esta tendencia, ya que las mujeres cuentan con más información para planear su vida familiar, académica y profesional (Welti, 2012). La transición epidemiológica también es consecuencia del éxito de las políticas de prevención y del acceso a los tratamientos de enfermedades infecciosas que son curables gracias al avance de los conocimientos médicos y la farmacología. Mientras que, en 1950, las principales causas de mortalidad en México correspondían principalmente a enfermedades infecciosas (gastroenteritis, gripe y enfermedades de la primera infancia), en 2013 prevalecían las enfermedades crónico-degenerativas como la diabetes mellitus, las enfermedades isquémicas del corazón y los tumores malignos (Soto-Estrada, Moreno-Altamirano y Pahua, 2016).

Si bien la creación de los sistemas públicos de salud en México comenzó a finales del siglo XIX, tomó forma después de la Revolución Mexicana. En 1917, se creó el Departamento de Salubridad Pública, que, en 1943, se fusionó con la Secretaría de Asistencia Pública y dio lugar a la Secretaría de Salubridad y Asistencia (SSA). Ese mismo año, el Presidente Manuel Ávila Camacho creó el Instituto Mexicano del Seguro Social (IMSS). Estas dos instituciones encauzaron el desarrollo sostenido del sistema mexicano de salud pública sobre la base de dos grandes pilares: uno dedicado a los trabajadores formales del sector privado, reservado al IMSS, y otro para la población sin cobertura de seguridad social contributiva, asignado a la SSA (Martínez, 2013). En 1960, con la fundación del Instituto de Seguridad y Servicios Sociales de los Trabajadores del Estado (ISSSTE), se creó un tercer pilar destinado a atender a los empleados gubernamentales.

Durante décadas, la estrategia del Estado mexicano consistió en que el acceso a la salud se produjera, cada vez en mayor medida, a través de los organismos de seguro social limitados a los trabajadores del sector formal de la economía; en particular, el IMSS y el ISSSTE. En consonancia con ese planteamiento, el sistema dedicado a atender a la población sin cobertura de seguridad social contributiva se concibió como un mecanismo de transición. Sin embargo, la dinámica poblacional impidió que se alcanzara este objetivo y, a partir de la crisis de la deuda y el cambio estructural de los años ochenta, el escaso crecimiento económico provocó que esa meta se fuera alejando a causa del crecimiento limitado del empleo formal. Por consiguiente, a principios del siglo XXI, México destinaba un bajo porcentaje del PIB al gasto público en salud, lo cual acarreaba consecuencias tanto en materia de bienestar demográfico como en lo que respecta a los principales indicadores sanitarios y de infraestructura.

Como puede apreciarse en el cuadro 1, México destaca en el contexto de la Organización de Cooperación y Desarrollo Económicos (OCDE) por ser uno de los países que menos gasto público destina como porcentaje del gasto total en salud de su economía: la proporción de gasto público es, junto con la de Turquía, una de las más bajas de los países de la muestra, y se encuentra por debajo del promedio de la OCDE e incluso de las cifras registradas en otros países de América Latina, como la Argentina, el Brasil, Colombia o el Perú (OPS, 2019). El gasto de los hogares representa el $41 \%$ del gasto en salud e incluye tanto el gasto en medicamentos, que supone una proporción importante, como los gastos catastróficos por emergencias de salud. En los países de la OCDE, el gasto público en sanidad alcanza, en promedio, un $71 \%$ del total del sector. Suecia y el Japón financian con recursos públicos el $84 \%$ del gasto en salud, mientras que Alemania, Francia, el Reino Unido y Turquía sufragan más del $75 \%$ con gasto público. Por otro lado, si bien los Estados Unidos destinan un porcentaje similar (un $50 \%$, frente al $51 \%$ de México), la magnitud del gasto público y la proporción respecto al tamaño de la economía son significativamente mayores en el caso estadounidense. 


\section{Cuadro 1}

México y países seleccionados de la Organización de Cooperación y Desarrollo Económicos (OCDE): indicadores del gasto en salud, 2008-2018

(En porcentajes)

\begin{tabular}{|c|c|c|c|c|c|c|c|c|c|c|}
\hline \multirow[b]{2}{*}{ Países } & \multirow{2}{*}{$\begin{array}{l}\text { Variación } \\
\text { porcentual } \\
\text { anual del } \\
\text { gasto en } \\
\text { salud per } \\
\text { cápita, } \\
\text { 2008-2018 }\end{array}$} & \multicolumn{5}{|c|}{$\begin{array}{l}\text { Sistema de financiamiento como } \\
\text { porcentaje del total, } 2018\end{array}$} & \multirow{2}{*}{$\begin{array}{c}\text { Porcentaje } \\
\text { del gasto } \\
\text { público } \\
\text { destinado } \\
\text { a la } \\
\text { salud, } \\
2018\end{array}$} & \multirow{2}{*}{$\begin{array}{c}\text { Gasto } \\
\text { en salud } \\
\text { procedente } \\
\text { de fuentes } \\
\text { públicas } \\
\text { como } \\
\text { porcentaje } \\
\text { del gasto } \\
\text { total en } \\
\text { salud, } 2018\end{array}$} & \multicolumn{2}{|c|}{$\begin{array}{l}\text { Inversión en } \\
\text { salud, } 2018\end{array}$} \\
\hline & & Otro & $\begin{array}{l}\text { Gasto } \\
\text { de los } \\
\text { hogares }\end{array}$ & $\begin{array}{c}\text { Seguro } \\
\text { de salud } \\
\text { voluntario }\end{array}$ & $\begin{array}{c}\text { Seguro } \\
\text { de salud } \\
\text { obligatorio }\end{array}$ & $\begin{array}{c}\text { Sistemas } \\
\text { gubernamentales }\end{array}$ & & & $\begin{array}{c}\text { Como } \\
\text { porcentaje } \\
\text { del gasto } \\
\text { total en } \\
\text { salud }\end{array}$ & $\begin{array}{c}\text { Como } \\
\text { porcentaje } \\
\text { del PIB }\end{array}$ \\
\hline Alemania & 2 & 2 & 13 & 1 & 78 & 6 & 20 & 78 & 9,6 & 1,1 \\
\hline Canadá & 2 & 2 & 15 & 13 & 1 & 68 & 19 & 73 & 5,6 & 0,6 \\
\hline Chile & 5 & & 34 & 6 & 58 & 2 & 17 & 50 & 2,7 & 0,2 \\
\hline España & 2 & 0 & 24 & 5 & 4 & 66 & 15 & 71 & 8,6 & 0,8 \\
\hline Estados Unidos & 3 & 4 & 11 & & 58 & 26 & 23 & 50 & 3,5 & 0,6 \\
\hline Francia & 1 & 1 & 9 & 7 & 78 & 5 & 15 & 77 & 5,1 & 0,6 \\
\hline Italia & 1 & 1 & 23 & 2 & & 74 & 13 & 74 & 4,1 & 0,4 \\
\hline Japón & 2 & 1 & 13 & 2 & 75 & 9 & 23 & 84 & 10,4 & 1,1 \\
\hline México & 1 & 2 & 41 & 6 & 28 & 24 & 11 & 51 & 1,3 & 0,1 \\
\hline OCDE & 2 & 2 & 21 & 4 & 37 & 36 & 15 & 71 & 5,6 & 0,5 \\
\hline Suecia & 2 & 1 & 15 & 1 & 0 & 84 & 19 & 84 & 5,1 & 0,6 \\
\hline Turquía & 3 & 5 & 17 & & 56 & 22 & 10 & 78 & 7,4 & 0,3 \\
\hline Reino Unido & 1 & 2 & 16 & 3 & 0 & 79 & 19 & 79 & 3,2 & 0,3 \\
\hline
\end{tabular}

Fuente: Organización de Cooperación y Desarrollo Económicos (OCDE), OECD Health Statistics 2020 [base de datos en línea] http://www.oecd.org/els/health-systems/health-data.htm.

Asimismo, México destaca como el país que menos invirtió en el sector de la salud en comparación con los demás Estados analizados: en 2018, apenas invirtió un 1,3\% del gasto total en salud, esto es, un $0,1 \%$ del PIB. Ese monto contrasta con las deficiencias en materia de infraestructura sanitaria que presenta el país, como salta a la vista en el cuadro 2. En un indicador tan importante como el número de camas de hospital por cada 10.000 habitantes, México se encuentra por debajo no solo de países desarrollados como Alemania y los Estados Unidos, sino también de países latinoamericanos como el Brasil y Chile. También lo superan otros países de ingreso medio, como Turquía. Este rezago se explica por la escasa inversión en infraestructura sanitaria realizada en las últimas décadas, que a su vez deriva de la insuficiente inversión en el sector de la salud desde los años ochenta hasta principios del siglo XXI.

Estas deficiencias se deben a que el gasto público en salud en México se ha mantenido por debajo del promedio de los países de la OCDE, e incluso del promedio latinoamericano. En los últimos 20 años, se distinguen dos tendencias claras en lo que respecta al gasto público en salud (antes y después de la crisis de 2008 y 2009): si bien en Europa y América Latina la tendencia era ascendente antes de la crisis, a partir de 2009 se registró un estancamiento en Europa y una desaceleración en América Latina (véase el gráfico 1). 


\section{Cuadro 2}

México y países seleccionados de la Organización de Cooperación y Desarrollo Económicos (OCDE): camas de hospital por cada 10.000 habitantes, 2019

(En número)

\begin{tabular}{llllccl}
\hline & Alemania & Brasil & Chile & Estados Unidos & México & Turquía \\
\hline 2008 & 82,13 & 24 & 21 & 32 & 16 & 23,39 \\
\hline 2009 & 82,42 & 24 & 21 & 31 & 16 & 23,98 \\
\hline 2010 & 82,50 & 24 & 20 & 30 & 17 & 25,20 \\
\hline 2011 & 82,24 & 23 & 21 & 30 & 15 & 25,34 \\
\hline 2012 & 81,64 & 23 & 22 & 29 & 15 & 26,61 \\
\hline 2013 & 82,78 & 23 & 22 & 29 & 16 & 26,56 \\
\hline 2014 & 82,78 & 22 & 22 & 29 & 15 & 26,66 \\
\hline 2016 & 83,00 & 22 & 22 & 29 & 15,2 & 27,00 \\
\hline 2017 & n.d. & n.d. & n.d. & n.d. & n.d. & n.d. \\
\hline 2018 & n.d. & n.d. & n.d. & n.d. & n.d. & n.d. \\
\hline
\end{tabular}

Fuente: Organización Mundial de la Salud (OMS), Global Health Expenditure Database (GHED), 2020 [en línea] https://apps.who. int/nha/database.

\section{Gráfico 1}

América Latina, Europa y México: gasto público en salud, 2000-2019

(En porcentajes del PIB)



Fuente: Organización Mundial de la Salud (OMS), Global Health Expenditure Database (GHED), 2020 [en línea] https://apps.who. int/nha/database.

En cambio, en las dos primeras décadas del siglo, en México se registró un crecimiento, si bien este no estuvo exento de algunas fluctuaciones. Dicho incremento resulta aún más significativo si se tiene en cuenta que se produjo en un período de lento crecimiento de la economía mexicana. A lo largo de poco más de un decenio, el aumento del gasto público en salud se mantuvo en términos reales. Sin embargo, a pesar de ese esfuerzo, México no logró alcanzar el promedio latinoamericano de gasto público en salud como porcentaje del PIB, y mucho menos el promedio europeo. A esto hay 
que añadir que el promedio latinoamericano tampoco cumple con los estándares recomendados a nivel internacional. De acuerdo con la Comisión Económica para América Latina y el Caribe (CEPAL), la mayoría de los países de la región no han invertido lo suficiente en el ámbito de la salud:

El gasto público del gobierno central en el sector, que en 2018 se situaba en un 2,2\% del PIB regional [...] está lejos del 6\% del PIB recomendado por la OPS para reducir las inequidades y aumentar la protección financiera en el marco del acceso y la cobertura universal. Los recursos adicionales podrían contribuir a fortalecer el primer nivel de atención, con énfasis en medidas de prevención (CEPAL, 2020a, pág. 10).

A lo largo de la historia reciente, el gasto público en salud ha registrado altibajos, lo cual refleja, a su vez, la política macroeconómica procíclica que ha predominado en México desde 1982 (Ros, 2013), en la que tanto el gasto social como las inversiones han sido las variables utilizadas para ajustar el gasto público en los momentos de crisis. Como puede apreciarse en el gráfico 2, el gasto público real en sanidad se contrajo en los contextos de crisis y desaceleración económica, como ocurrió durante la crisis de la deuda de 1982, que se vio agudizada en 1986 por la caída de los precios del petróleo. Después de un período de recuperación a finales de los ochenta y principios de los noventa, se produjo una nueva disminución a partir de 1993, que se vio agravada por la crisis de 1995. A partir de 1996, se registró una recuperación que se mantuvo incluso en 2009, cuando se hicieron ajustes en algunos rubros del gasto social (educación y salud, principalmente) y la inversión (infraestructura y energía). Esa medida coincidió con el mayor esfuerzo jamás desplegado por el Gobierno mexicano para atender a la población sin cobertura de seguridad social contributiva, que, lejos de disminuir, iba en aumento a causa de la contracción del empleo en el sector formal de la economía. Sin embargo, a partir de 2012, se observó una clara desaceleración en ese crecimiento.

\section{Gráfico 2}

México: gasto público en salud, 1980-2019

(En miles de millones de pesos mexicanos de 2018)

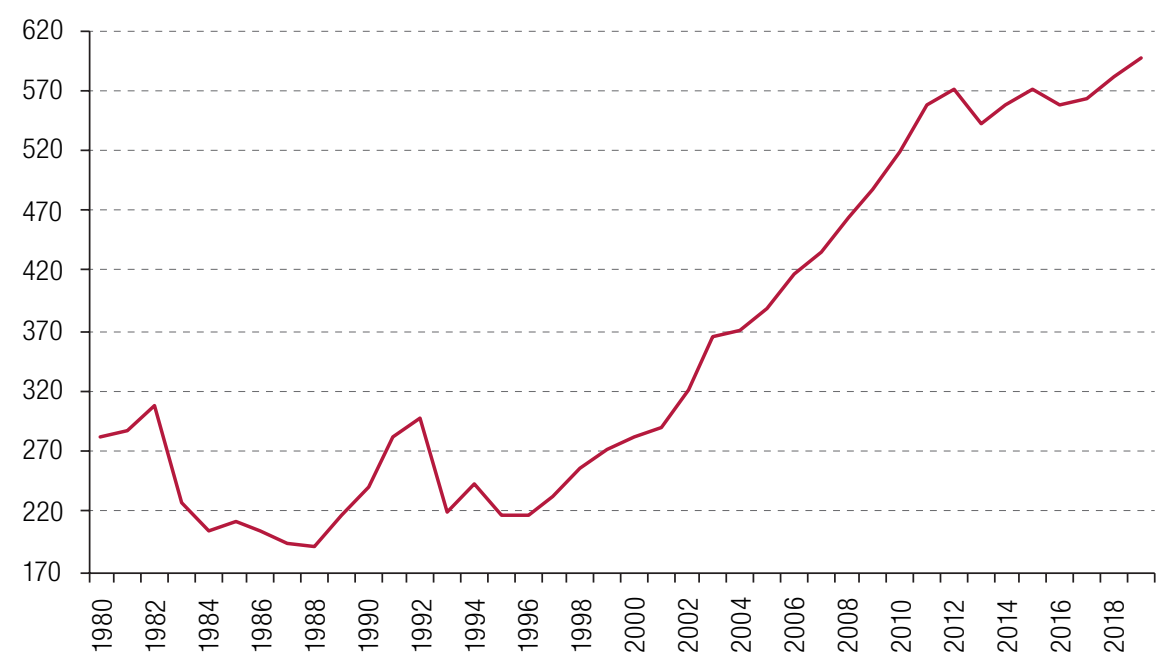

Fuente: Instituto Nacional de Estadística y Geografía (INEGl), "Salud", 2020 [base de datos en línea] https://www.inegi.org.mx/ temas/saludsat/.

Es indudable que México debe destinar más recursos públicos a la salud. El elevado porcentaje del gasto que recae en los hogares es un reflejo de la importancia que sigue teniendo el acceso a esas prestaciones en el bienestar de la población, así como de sus efectos en la pobreza y la desigualdad. La falta de acceso a los servicios de salud públicos expone a la población que recibe ingresos 
insuficientes y precarios a caer en una situación de pobreza ante posibles cuadros de enfermedad que entrañen gastos catastróficos. Al mismo tiempo, la segmentación de los sistemas, la oferta diferenciada de prestaciones y las brechas en la calidad de la atención recibida son factores que contribuyen a profundizar la desigualdad económica y social característica de México.

Un sistema público de salud con cobertura universal y una oferta integral de prestaciones ayudaría notablemente a reducir el riesgo de pobreza, disminuir la desigualdad y fortalecer la cohesión social. Para que un sistema así sea viable, debe financiarse con impuestos generales, y esto solo es sostenible a largo plazo si se emprende una profunda reforma fiscal redistributiva. La ironía es que, de no avanzar hacia la construcción de ese sistema (una meta que, en principio, parece ambiciosa), la segmentación puede resultar más cara a largo plazo, tanto en términos económicos como de ampliación de brechas entre la población sin cobertura de seguridad social contributiva y la población derechohabiente de alguno de los organismos de seguridad social.

\section{La microeconomía de la salud}

De acuerdo con la Cuenta Satélite del Sector Salud de México de 2018, ese año la aportación del sector sanitario equivalió al 5,7\% del PIB de México; un 4,1\% correspondió al PIB de las actividades económicas del sector de la salud y un 1,6\%, al valor del trabajo no remunerado relativo a la atención de la salud. En cuanto al PIB de las actividades económicas del sector, el 71,9\% obedeció a las unidades económicas que lo integran, mientras que el 28,1\% correspondió al valor monetario del trabajo no remunerado realizado por los hogares en lo que respecta al cuidado de los enfermos. En cuanto al origen de los bienes requeridos por el sector de la salud, el 80,2\% fueron de procedencia nacional, el $10,4 \%$ se importaron y el 9,4\% restante correspondió al margen de comercio y distribución. El saldo de la balanza de bienes y servicios del sector fue favorable, y se registraron exportaciones netas por valor de 11.799 millones de pesos. Entre ellas, destacó la exportación de materiales para vendaje y curación de heridas y otros bienes para el cuidado de la salud. Ese mismo año, el sector sanitario generó 2.204.897 nuevos puestos de trabajo remunerados, lo que representa un 5,1\% del empleo total a nivel nacional (INEGl, 2019).

Es preciso señalar que, si bien la balanza comercial y de servicios del sector de la salud es superavitaria, no ocurre lo mismo en el sector de la fabricación de medicamentos, directamente relacionado. La industria farmacéutica mexicana ha sufrido altibajos desde la crisis de 2009, que han quedado reflejados en una disminución de su participación en el PIB manufacturero, del 5,2\% en 2003 al 3,2\% en 2014. Esa reducción se ha debido, entre otros factores, a las fluctuaciones del tipo de cambio, ya que aproximadamente la mitad de sus insumos son importados, lo que se ha traducido en un incremento de las importaciones. La importación de medicamentos e insumos farmacéuticos ascendió a 70.607 millones de pesos en 2018, mientras que las exportaciones sumaron 22.143 millones de pesos. Ese año, el saldo de la balanza comercial ascendió a 48.464 millones de pesos. En lo que respecta a las importaciones del sector de la salud, destaca la importación de equipos para el cuidado de la salud, materiales para vendaje y curación de heridas, y lentes (INEGl, 2019).

Ante las debilidades que ha puesto de manifiesto la actual crisis sanitaria, la política industrial debería dar prioridad al sector farmacéutico y al sector de la producción de equipos de seguridad y tecnología para la atención de la salud. Como señaló la Secretaria Ejecutiva de la CEPAL, Alicia Bárcena, en una reunión informativa de los países miembros de la Conferencia de Ciencia, Innovación y Tecnologías de la Información y las Comunicaciones (un órgano subsidiario de la CEPAL): "Tenemos que acercar la ciencia, la tecnología y la innovación a los sectores productivos [...] como es el caso de la fabricación de insumos médicos, de diversos productos de protección sanitaria, de exámenes para detectar el virus y de equipos médicos críticos como, por ejemplo, los ventiladores mecánicos, entre 
otros" (CEPAL, 2020c). En momentos de crisis como el vivido en 2020, la reducción de la dependencia nacional de los medicamentos y equipos esenciales para la atención de la salud adopta un carácter estratégico, al tiempo que abre nuevas posibilidades de desarrollo industrial, innovación tecnológica y sustitución de importaciones para nuestros países.

\section{Los retos de México para garantizar el derecho a la salud}

A pesar de los avances logrados por México en lo que respecta a la esperanza de vida, el estado de la salud en el país no es satisfactorio, ya que el sistema de salud pública presenta deficiencias importantes en tres indicadores centrales: igualdad, calidad y cobertura financiera, como se observa en el cuadro 3.

\section{Cuadro 3}

México y Organización de Cooperación y Desarrollo Económicos (OCDE): indicadores de igualdad, calidad y cobertura financiera, 2019

\begin{tabular}{lcc}
\hline & México & OCDE \\
\hline Igualdad & & \\
\hline Cobertura de los servicios de salud (en porcentajes) & 89,3 & 98,4 \\
\hline Protección financiera (en porcentajes) & 51,3 & 71,0 \\
\hline Calidad & 85 & 225 \\
\hline Efectividad de los servicios básicos & 27,5 & 6,9 \\
\hline Efectividad de los servicios secundarios & & \\
\hline Cobertura financiera & 1138 & 3994 \\
\hline Gasto en salud per cápita (en dólares) & 5,5 & 8,8 \\
\hline Gasto en salud (como porcentaje del PIB) & &
\end{tabular}

Fuente: Organización de Cooperación y Desarrollo Económicos (OCDE), Health at a Glance 2019: OECD Indicators, París, 2019.

Nota: La cobertura de los servicios de salud se calcula como el porcentaje de la población que cumple los requisitos para acceder a servicios básicos; la protección financiera es el porcentaje de la población mayor de 15 años cuyo gasto en salud es cubierto por fuentes públicas; la efectividad de los servicios básicos se desprende de la cantidad de admisiones hospitalarias por asma y enfermedad pulmonar obstructiva crónica (EPOC) por cada 100.000 habitantes, y la efectividad de los servicios secundarios, de la mortalidad a 30 días por infarto agudo de miocardio por cada 100.000 habitantes.

Estas deficiencias no solo destacan en las comparaciones internacionales, sino que se agudizan en el propio país. Las desigualdades y los contrastes continúan siendo una de las principales características de México, y se reproducen en las esferas del acceso a la salud y la infraestructura sanitaria, tanto a nivel de las personas y los grupos sociales como en el plano regional. Se puede argumentar que, dado que el nivel de ingresos de un país determina las condiciones de su sector sanitario, México podrá aspirar a mejores niveles de salud en la medida en que mejore su desempeño económico. Sin embargo, diversos estudios demuestran que el nivel de la salud también repercute de forma directa en el crecimiento económico. Como han señalado recientemente la CEPAL y la Organización Panamericana de la Salud (OPS): "el crecimiento económico sostenible es un componente central de la salud y el bienestar integral de las personas, a la vez de proteger y promover la salud de la población debe ser una piedra angular y una apuesta estratégica para el crecimiento y el desarrollo a largo plazo" (CEPAL/ OPS, 2020, pág. 18).

En el caso de México, en una investigación de David Mayer-Foulkes (Mayer-Foulkes, 2001) sobre la relación entre la esperanza de vida y la tasa de mortalidad (en diferentes grupos de edad) y el crecimiento 
económico, se sostiene que los avances en materia de salud contribuyeron a aproximadamente un tercio del crecimiento (potencial) registrado en México entre 1970 y 1995. En todo caso, el crecimiento de la población y de la esperanza de vida a lo largo del siglo XX permitió pasar de 13 millones de habitantes en 1900 a más de 100 millones en 2000 y, en dos décadas (1940 a 1960), México pasó de ser un país abrumadoramente rural a convertirse en uno predominantemente urbano (Welti, 2012). Es indudable que esta gran transformación no se habría logrado sin las inversiones en salud de los Gobiernos posrevolucionarios, aunque resultaran insuficientes.

El desarrollo del sistema se produjo de forma simultánea a la industrialización y urbanización aceleradas que caracterizaron las décadas que siguieron a la Revolución Mexicana. Esto también permitió que se difundieran más fácilmente las medidas de higiene general y los tratamientos que atendían, a un bajo costo, las enfermedades infecciosas más comunes. Esos avances facilitaron el incremento de la esperanza de vida al nacer y, gracias a ellos, en 1950 ya se percibían progresos notables en la disminución de las tasas de mortalidad materno-infantil, lo que repercutió en el crecimiento demográfico. La única discontinuidad en una pirámide de edades casi perfecta, de acuerdo con el censo de ese año, se observa en el grupo de los nacidos durante los años más críticos de la Revolución Mexicana (1913-1916) y la gripe española (1918-1919) (véase el gráfico 3).

\section{Gráfico 3}

México: pirámide poblacional, 1950

(En porcentajes)

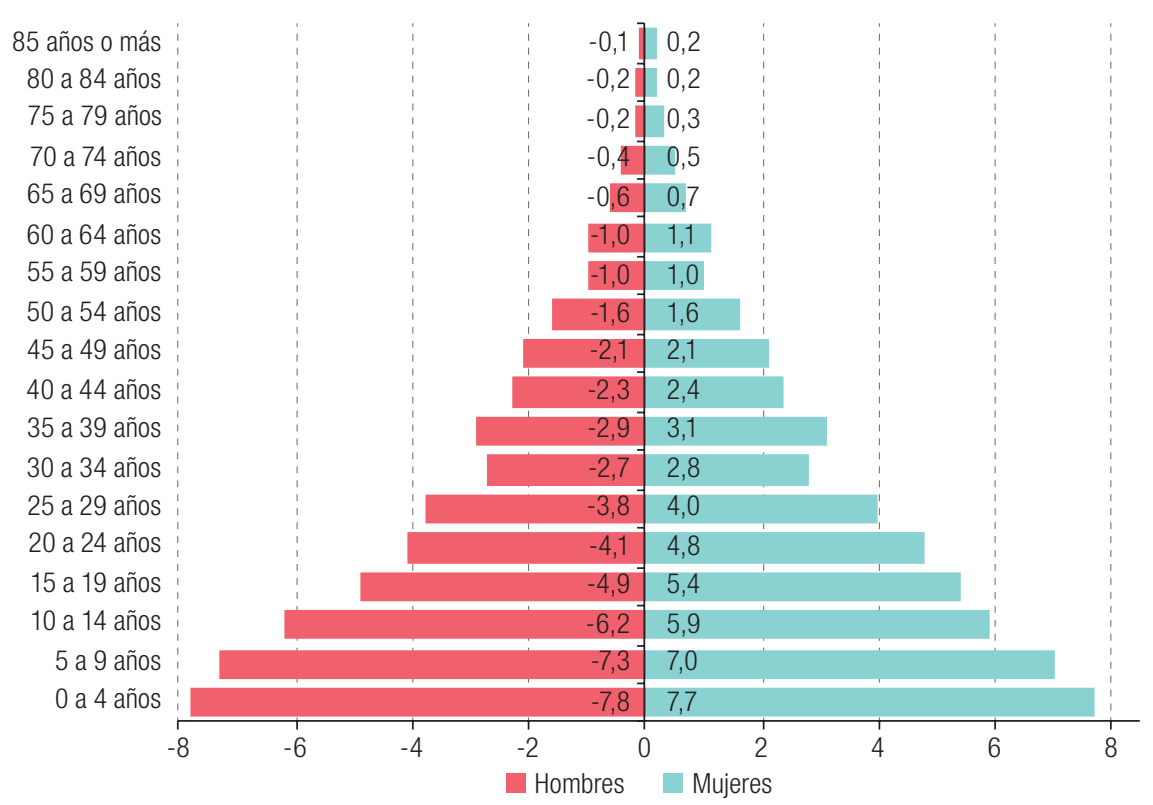

Fuente: Instituto Nacional de Estadística y Geografía (INEGI), Séptimo Censo General de Población, Aguascalientes, 1950.

La política social del Estado posrevolucionario, basada en incorporar a la población a un sistema de protección sanitaria mediante la afiliación de los derechohabientes y sus familias a los organismos de seguridad social, entró en crisis en los años ochenta. Ya antes se había hecho evidente la existencia de un sector de la economía informal que era difícil de absorber por esos organismos, pero, a partir de entonces, los vaivenes de la economía y sus efectos en el empleo formal provocaron que se estancara el crecimiento de la cobertura. A esto hay que añadir el deterioro en la calidad, derivado de las políticas de ajuste emprendidas en los años ochenta y del incremento en los costos de la atención sanitaria. Esta situación coincidió con un momento de la transición demográfica caracterizado por el aumento 
de la población en edad de trabajar y la disminución de la población dependiente menor de quince años, lo que dio lugar a un bono demográfico que se ha desaprovechado a causa del lento crecimiento de la economía mexicana. Ante la incapacidad de generar suficientes empleos en el sector formal, las principales válvulas de escape fueron la emigración y la informalidad (Lomelí y Vázquez, 2016).

México pasó de una estructura poblacional predominantemente infantil y juvenil en 1950 a otra muy distinta en 2015, gracias al éxito de la política poblacional iniciada en 1974 en virtud de la Ley General de Población promulgada ese año, la creación del Consejo Nacional de Población (CONAPO) y el inicio sistemático de campañas de planificación familiar. Esas políticas han redundado en una disminución de la tasa de crecimiento de la población, que se ha reflejado en la reducción de la base de la pirámide demográfica, el consiguiente aumento de la población en edad de trabajar y la disminución del coeficiente de dependencia. Estos cambios son fácilmente observables en la pirámide poblacional de 2015, a pesar del repunte de la tasa de natalidad registrado en la primera década del siglo XXI (véase el gráfico 4).

\section{Gráfico 4}

México: pirámide poblacional, 2015

(En porcentajes)

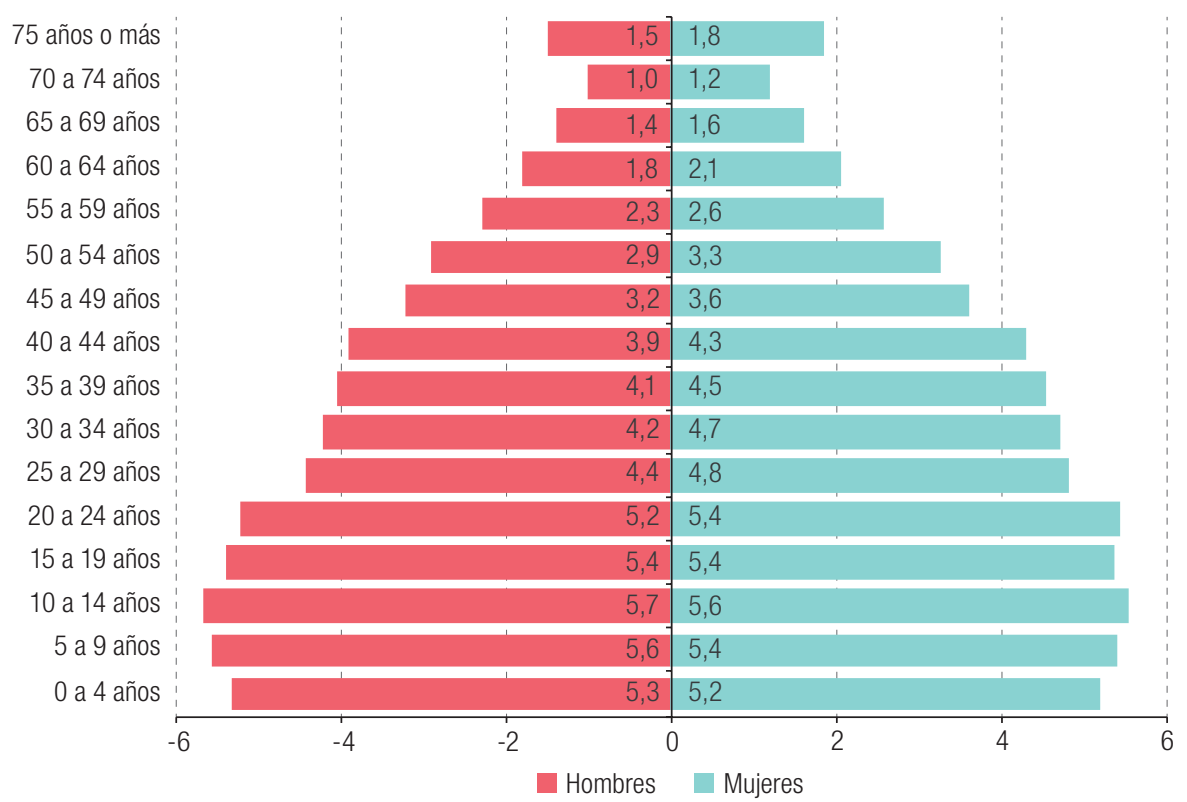

Fuente: Instituto Nacional de Estadística y Geografía (INEGI), Encuesta Intercensal 2015 [base de datos en línea] https://www. inegi.org.mx/programas/intercensal/2015/default.html

No obstante, pese a las reformas emprendidas y el incremento del gasto per cápita en salud entre 1997 y 2007, a una tasa promedio real anual del 4,4\% (superior al promedio de 4,1\% registrado en los países de la OCDE), México presenta asimetrías entre la atención prestada por el sistema de salud y las necesidades de la población. Debido a la crisis ocurrida en los años ochenta y sus secuelas en los años noventa, se observa que, a pesar de los avances alcanzados en el siglo XX, persisten algunas deficiencias, a las que se han sumado los nuevos retos derivados de las transiciones demográfica y epidemiológica. En conjunto, estas fallas contribuyen a mantener $-\mathrm{y}$ ahondar - los problemas derivados no solo de la desarticulación del sistema de salud (instituciones y programas), sino también de la cobertura insuficiente y desigual, la baja eficacia en la asignación de los recursos, la mala utilización de las unidades médicas y los desequilibrios financieros. A estos problemas se suma la distribución desigual de la infraestructura sanitaria en el territorio nacional, que reproduce en 
gran medida las desigualdades regionales y que, en términos generales, coincide con la distribución geográfica de la pobreza (Lomelí, Flores y Granados, 2012).

En 2016, el gasto público representó el 52\% del total del gasto en salud y, con esos recursos, se financió el funcionamiento de los dos tipos básicos de instituciones: las que ofrecen servicios a la población derechohabiente por su condición laboral (IMSS, ISSSTE, los servicios de salud de Petróleos Mexicanos (PEMEX), la Secretaría de la Defensa Nacional (SEDENA) y la Secretaría de Marina) y los programas dedicados a atender a la población que carece de seguridad social. Ese mismo año, el gasto privado ascendió al $48 \%$, del cual más del $80 \%$ correspondió a gasto de bolsillo y el resto, a sistemas de aseguramiento privado. Más allá de las comparaciones internacionales y la falta de parámetros universales acerca del monto óptimo de la inversión en salud con respecto al tamaño de una economía, conviene preguntarse si la inversión de México es la adecuada (considerando el gasto total, las fuentes de financiamiento y su distribución). La pandemia ha puesto en evidencia que la inversión en salud no solo es inferior a la requerida en un país con el nivel de desarrollo y las necesidades de México, sino que su distribución no contribuye a reducir las disparidades en los indicadores de salud.

El incremento, en términos reales, de los recursos federales asignados a la población que carece de seguridad social no ha logrado equilibrar la disparidad entre el gasto público destinado a la población asegurada y el destinado a la no asegurada. En 2004, entró en funcionamiento el Seguro Popular, cuyo financiamiento era teóricamente tripartito: recursos federales asignados por la Secretaría de Salud, recursos estatales y cuota familiar, de la cual estaban exentas las familias que pertenecían al 40\% de los hogares más pobres. En 2009, los recursos de que dispuso el Seguro Popular supusieron el 48,6\% del gasto total del ramo 12 (salud) del Presupuesto de Egresos de la Federación y ascendieron a 41.368 millones de pesos, un 5,7\% más que en 2008. En 2019, el Seguro Popular recibió 80.144 millones, que representaron el $64,5 \%$ de los recursos asignados al ramo 12 . Ese aumento considerable de los recursos permitió que, en 2017, el gasto público supusiera el $52 \%$ del gasto total en salud. Sin embargo, el gasto per cápita total de las personas que carecían de seguridad social fue de 3.954,9 pesos mexicanos, en comparación con los 5.644,7 pesos de quienes contaban con seguridad social, lo cual refleja una vez más los problemas de desigualdad derivados de la segmentación de los sistemas públicos (CONEVAL, 2020).

En noviembre de 2019, el gobierno federal anunció la desaparición del Seguro Popular y su incorporación al Instituto de Salud para el Bienestar (INSABI). Como se explica en su página institucional (INSABI, 2020), el INSABI tiene como propósito proporcionar "servicios de salud gratuitos y de calidad a todas las personas que se encuentren en el país y no cuenten con seguridad social", bajo "criterios de universalidad, igualdad e inclusión". Además, "los beneficiarios del INSABI recibirán servicios médicos sin restricciones porque habrá atención universal para todos los padecimientos, incluidos aquellos que generan gastos catastróficos". También contempla "garantizar y mejorar la atención en los servicios públicos de salud", así como asegurar "el abasto de medicamentos y el equipamiento suficiente para la atención de los beneficiarios en todos los niveles de atención a la salud" y, además, se "rehabilitará y ampliará la infraestructura médica".

Resulta innegable que es preciso seguir ampliando los servicios de salud y lograr una adecuada integración y articulación de los programas públicos. Sin embargo, no es evidente que la cancelación del Seguro Popular y la creación del INSABI sean la solución más conveniente para ampliar la cobertura. Más aún, cabe preguntarse si la creación de un nuevo programa no contribuye a la segmentación de los servicios públicos, la heterogeneidad de sus prestaciones y la ineficiencia operativa del sistema nacional de salud. Sin duda, la prioridad presupuestal dada a este nuevo organismo, junto con el diseño organizacional e institucional adecuado, son requisitos indispensables para que cumpla su propósito fundacional.

El tema de la cobertura no es banal en un país que se enfrenta al envejecimiento progresivo de 
su población. Esto, que aún no es un problema, lo será dentro de tres décadas. De mantenerse las actuales tendencias demográficas, a mediados de siglo, el porcentaje de adultos mayores de 60 años que precisen servicios de salud y no estén cubiertos por la seguridad social se habrá incrementado significativamente. Como se desprende fácilmente del análisis plasmado en el gráfico 5 , desde los años sesenta, el grupo poblacional menor de 15 años ha venido decreciendo aceleradamente, al tiempo que, desde los ochenta, los mayores de 30 años se han incrementado de manera acelerada. Esta tendencia apunta a un envejecimiento progresivo de la población, que entrañará la necesidad de destinar mayores recursos tanto a la salud como a la seguridad social a mediados del siglo XXI.

\section{Gráfico 5}

México: evolución de la distribución poblacional por rango etario, 1950-2015

(En porcentajes del total)



Fuente: Instituto Nacional de Estadística y Geografía (INEGI), Encuesta Intercensal 2015 [base de datos en línea] https://www. inegi.org.mx/programas/intercensal/2015/default.html.

Las tendencias demográficas apuntan al envejecimiento de la población, mientras que la transición epidemiológica en curso conduce también al incremento de los costos de la atención sanitaria. La aparición de nuevas afecciones - en particular, las derivadas de la propagación o la rápida mutación de virus como el SARS-CoV-2 - requerirá una mayor inversión en investigación en el ámbito de la salud y el desarrollo de nuevos mecanismos de prevención y atención, así como el fortalecimiento de las capacidades de los servicios de medicina curativa. De ahí que los retos de aumentar la cobertura, reorganizar las prioridades del sistema y mejorar la calidad de la atención estén interrelacionados.

A las consideraciones anteriores hay que añadir que el acceso a los servicios de salud sigue siendo un factor que incide en la pobreza y desigualdad de una proporción considerable de la población mexicana. De acuerdo con el Consejo Nacional de Evaluación de la Política de Desarrollo Social (CONEVAL, 2020), a partir de los datos recabados en la Encuesta Nacional de Ingresos y Gastos de los Hogares 2018, ese año había en México 20,2 millones de personas sin acceso a los servicios de salud, y 71,7 millones no tenían acceso a la seguridad social. Ese dato resulta interesante, porque la población no afiliada a los organismos de seguridad social contaba con la cobertura del Seguro Popular, que incluía un menor número de intervenciones médicas (294 según su último catálogo, de septiembre de 2019) (CNPSS, 2019) que la de los afiliados al IMSS y al ISSSTE. 
Aun así, ni siquiera los datos anteriores revelan toda la problemática de la salud en México. Según el INEGI, en 2020 la población afiliada a los organismos de seguridad social o a los servicios públicos de salud para la población sin cobertura de seguridad social contributiva asciende al 82,2\% del total, mientras que el 23,1\% de la población recurre a servicios médicos privados (INEGI, 2019). Ese último porcentaje abarca tanto a quienes pueden pagar la medicina privada porque su nivel socioeconómico lo permite como a los que recurren a ella en situaciones de emergencia porque, pese a ser derechohabientes, no quieren o no pueden esperar su turno para ser atendidos por las instituciones públicas. En efecto, ante la falta de recursos, los tiempos de espera se han convertido en el principal mecanismo de racionamiento empleado por los servicios públicos de salud para administrar el exceso de demanda ante una oferta insuficiente. El reto del INSABI consiste no solo en aumentar la cobertura, sino también la cantidad de intervenciones médicas cubiertas y la capacidad de la atención prestada, de modo que se reduzcan los tiempos de espera.

Tal como ha quedado demostrado en la emergencia sanitaria de 2020, un último aspecto importante que es preciso considerar es el carácter estratégico de sustituir las importaciones de medicamentos e insumos médicos. De acuerdo con la CEPAL, "la disrupción de diversas cadenas globales de valor ha mostrado los riesgos que supone la elevada dependencia regional de las manufacturas importadas. Esto ha quedado particularmente de manifiesto con las severas limitaciones a la disponibilidad de productos esenciales para el combate al COVID-19 a raíz de las restricciones impuestas por la mayoría de sus principales proveedores" (CEPAL, 2020b, pág. 19).

\section{Conclusiones}

El principal reto para garantizar el derecho a la salud consiste en universalizar la cobertura sin provocar nuevas asimetrías por la heterogeneidad en la calidad y la oferta de las prestaciones de los sistemas públicos de salud, derivada, a su vez, de la segmentación. Para que el INSABI logre su objetivo, será necesario dotarlo de suficientes recursos materiales, financieros y humanos y de una organización adecuada para que funcione en beneficio de la población sin cobertura de seguridad social contributiva a corto plazo y, a la vez, sea flexible a largo plazo, con miras a promover su integración y articulación con las demás instituciones públicas del sector, a fin de construir un auténtico sistema nacional de salud.

No hay, ni puede haber, desarrollo humano sin un sistema de salud universal e integral. El Estado no puede abdicar de su responsabilidad de garantizar el acceso a la salud a todos los ciudadanos. Para ello, es preciso desplegar un esfuerzo fiscal que permita financiar un sistema nacional de salud de esas características. El mercado se limitará a reproducir las asimetrías de la distribución del ingreso en el acceso a los servicios de salud. Bajo esa óptica, es evidente que las fuerzas del mercado o las negociaciones comerciales no son, ni pueden ser, las que determinen los niveles de salud de la población. La elaboración de las políticas que inciden en la salud de los mexicanos constituye una tarea ineludible del Estado y, lo que es más, se trata de una obligación consagrada en la Constitución Política de México, que en su artículo 4 reconoce el derecho a la salud. Es responsabilidad de todos -y, en particular, de los legisladores - diseñar los mecanismos adecuados para garantizar ese derecho.

\section{Bibliografía}

CEPAL (Comisión Económica para América Latina y el Caribe) (2020a), "América Latina y el Caribe ante la pandemia del COVID-19: efectos económicos y sociales", Informe Especial COVID-19, № 1, Santiago, abril. (2020b), "Dimensionar los efectos del COVID-19 para pensar en la reactivación", Informe Especial COVID-19, № 2, Santiago, abril. 
(2020c), "Aporte de la ciencia, tecnología e innovación es clave para enfrentar los desafíos en la industria de la salud y la recuperación económica tras la pandemia", Santiago, 7 de mayo [en línea] https://www.cepal. org/es/comunicados/aporte-la-ciencia-tecnologia-innovacion-es-clave-enfrentar-desafios-la-industria-la.

CEPAL/OPS (Comisión Económica para América Latina y el Caribe/Organización Panamericana de la Salud) (2020), "Salud y economía: una convergencia necesaria para enfrentar el COVID-19 y retomar la senda hacia el desarrollo en América Latina y el Caribe", Informe COVID-19 CEPAL-OPS, Santiago, julio.

CNPSS (Comisión Nacional de Protección Social en Salud) (2019), "Catálogo Universal de Servicios de Salud (CAUSES) 2019”, Ciudad de México, septiembre [en línea] https://www.gob.mx/salud/seguropopular/ articulos/catalogo-universal-de-servicios-de-salud-causes-2019.

CONEVAL (Consejo Nacional de Evaluación de la Política de Desarrollo Social) (2020), La política social en el contexto de la pandemia por SARS-CoV-2 (COVID-19) en México, Ciudad de México.

Deaton, A. (2015), El gran escape: salud, riqueza y los orígenes de la desigualdad, Ciudad de México, Fondo de Cultura Económica (FCE).

INEGI (Instituto Nacional de Estadística y Geografía) (2019), "Cuenta Satélite del Sector Salud de México, 2018", Comunicado de Prensa, № 690/19, Aguascalientes.

(2017), Estadísticas a propósito de... la industria farmacéutica y sus proveedores, Aguascalientes.

INSABI (Instituto de Salud para el Bienestar) (2020), "Instituto de Salud para el Bienestar" [en línea] https:// www.gob.mx/insabi/articulos/instituto-de-salud-para-el-bienestar-230778.

Lomelí, L. y L. Vázquez (2016), "Cambio estructural y migración: el caso de México", Economía UNAM, vol. 13, № 39, Ciudad de México, Universidad Nacional Autónoma de México (UNAM).

Lomelí, L., C. Flores y V. Granados (2012), "Niveles de vida, desigualdad y pobreza", Los determinantes sociales de la salud en México, R. Cordera y C. Murayama (coords.), Ciudad de México, Fondo de Cultura Económica/Universidad Nacional Autónoma de México (FCE/UNAM).

Martínez, À. (2014), "La economía de la salud y la salud de la economía", El farmacéutico, № 500, Barcelona, Ediciones Mayo, febrero.

Martínez, G. (2013), Un sistema en busca de salud: desarrollo, declive y renovación del sistema de salud mexicano, Ciudad de México, Fondo de Cultura Económica (FCE).

Mayer-Foulkes, D. (2001), "The long-term impact of health on economic growth in Mexico, 1950-1995", Journal of International Development, vol. 13, № 1, Hoboken, Wiley.

OCDE (Organización de Cooperación y Desarrollo Económicos) (2019), Health at a Glance 2019: OECD Indicators, París.

(2009), Health at a Glance 2009: OECD Indicators, París.

(2005), Estudios de la OCDE sobre los sistemas de salud: México, París, junio.

OPS (Organización Panamericana de la Salud) (2019), Indicadores básicos 2019: tendencias de la salud en las Américas, Washington, D.C.

Ros, J. (2013), Algunas tesis equivocadas sobre el estancamiento económico de México, Ciudad de México, El Colegio de México/Universidad Nacional Autónoma de México (COLMEX/UNAM).

Soto-Estrada, G., L. Moreno-Altamirano y D. Pahua (2016), "Panorama epidemiológico de México: principales causas de morbilidad y mortalidad", Revista de la Facultad de Medicina, vol. 59, № 6, Ciudad de México, Universidad Nacional Autónoma de México (UNAM).

Welti, C. (2012), "Transición demográfica y salud en México", Los determinantes sociales de la salud en México, R. Cordera y C. Murayama (coords.), Ciudad de México, Fondo de Cultura Económica/Universidad Nacional Autónoma de México (FCE/UNAM). 